\title{
Spots and clots - leave them or treat them? Why and how to treat
}

\author{
Dennis M Jensen MD
}

\begin{abstract}
DM Jensen. Spots and clots - leave them or treat them? Why and how to treat. Can J Gastroenterol 1999;13(5):413-415. The clinical indication for urgent endoscopy with combined diagnosis and treatment is bleeding that is severe enough to seek medical attention. The author uses stigmata of ulcer hemorrhage as a guide to endoscopic therapy. Active arterial bleeding, nonbleeding visible vessels and adherent nonbleeding clots are always treated endoscopically. In randomized trials, patients have demonstrated better outcomes from endoscopic therapies than from medical therapies. Flat spots, grey or black sloughs, and clear ulcer bases are not treated endoscopically. The clinical condition and the endoscopic appearance of the ulcer (ie, stigmata of hemmorrhage) of the patient with upper gastrointestinal bleeding are used to determine the subsequent level of care (discharge, ward or intensive care).
\end{abstract}

Key Words: Endoscopy; Stigmata; Ulcer hemmorrhage

\section{Taches et caillots - les laisser ou les traiter? Pourquoi et comment les traiter}

RÉSUMÉ : L'indication clinique pour une endoscopie d'urgence associée à un diagnostic et à un traitement est un saignement suffisamment sévère pour consulter un médecin. L'auteur utilise les stigmates d'une hémorragie d'origine ulcéreuse comme guide de traitement endoscopique. Un saignement artériel actif, des vaisseaux apparents sans saignement et des caillots adhérents sans saignement sont toujours traités par endoscopie. Dans les essais randomisés, les patients ont démontré des résultats meilleurs à la suite des traitements par endoscopie comparativement à des traitements médicaux. Les taches plates, les escarres noires ou grises, et des bases claires d'ulcère ne sont pas traitées par endoscopie. L'état clinique et l'apparence endoscopique de l'ulcère (c'est-à-dire, les stigmates d'hémorragie) du patient accusant un saignement des voies gastro-intestinales supérieures servent à déterminer le niveau subséquent des soins (congé, salle ou soins intensifs).
$\mathrm{T}$ he clinical indication for urgent endoscopy with combined diagnosis and treatment is bleeding that is severe enough to present for medical attention. The CURE Hemostasis Group uses stigmata of ulcer hemorrhage as a guide to endoscopic therapy. Specifically, the following stigmata are always treated endoscopically: active arterial bleeding, nonbleeding visible vessels and adherent nonbleeding clots. Figure 1 shows the rebleeding rate of ulcers with different stigmata treated with medical therapy.

The rationale for endoscopic treatment is that, in randomized trials, patients treated by the CURE Hemostasis Group have demonstrated significantly better outcomes from endoscopic therapies (heater probe, bipolar, or combination injection and thermal) than from medical therapies (1-3). Persistent oozing without other stigmata is also treated, although this is not a very prevalent lesion, and in the absence of coagulopathies an advantage over medical therapy is difficult to document. The following are not treated endoscopically: flat spots, sloughs (grey or black) and clear ulcer bases. For such minor stigmata of ulcer hemorrhage, randomized trials have not shown a significant improvement over medical therapy (3), and in some older laser trials complications of therapy (such as induction of bleeding) were frequent $(1,2)$ (Tables 1,2$)$.

Most patients with ulcer hemorrhage start bleeding out of the hospital, and minor stigmata of ulcer hemorrhage are more prevalent. Figure 2 shows the prevalence of stigmata in large CURE studies.

In contrast, patients who develop hemorrhage after hospitalization for an unrelated problem (such as surgery or a comorbid illness) more often have major stigmata of ulcer hemorrhage and require endoscopic therapy $(1,2)$.

UCLA School of Medicine, Los Angeles, California, USA

Correspondence and reprints: Dr Dennis M Jensen, Professor of Medicine, UCLA School of Medicine, 44-133 CHS, GI Division, 10833 Le Conte Avenue, Los Angeles, California 90024-1684, USA. Telephone 310-478-3711 ext 43324, fax 310-825-1700,

e-maildjensen@med1.medsch.ucla.edu 


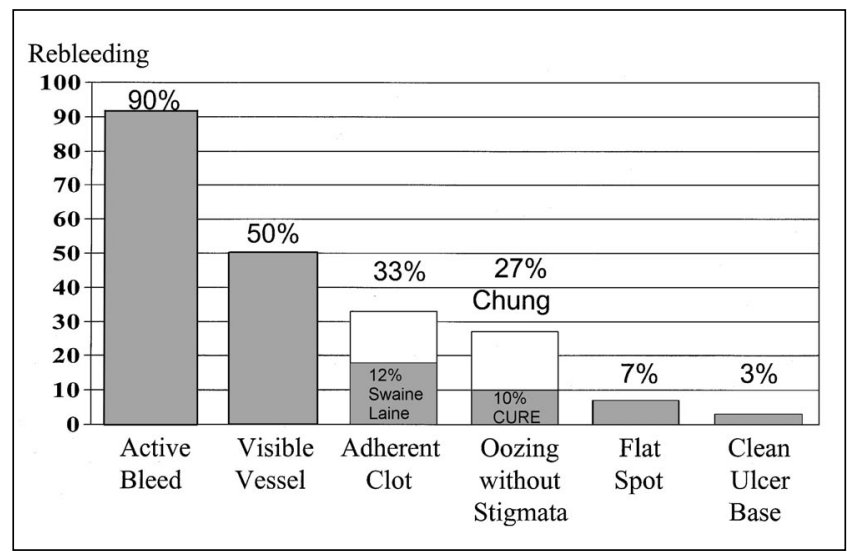

Figure 1) Natural history of ulcer hemorrhage. Data from references 1,2

TABLE 1

Management of a flat spot on an ulcer after severe upper gastrointestinal hemorrhage

- Stigma are verified by vigorous washing and tangential views

- The rebleeding rate is $\leq 7 \%$ with medical therapy

- No benefit of endoscopy treatments has been shown in randomized, controlled trials

- The spot is biopsied for Helicobacter pylori

- The patient is re-fed early and discharged if reliable

TABLE 2

Management of a clean ulcer base found after severe upper gastrointestinal bleeding

- The rebleeding rate is $\leq 3 \%$ with medical therapy

- Target irrigation of the ulcer base is performed to remove exudate and to rule out another stigmata (such as a spot or visible vessel)

- Helicobacter pylori-positive patients are treated

- Health services studies confirm the safety and reduce the costs of same-day discharge

- Patients are counselled about acetylsalicylic acid and nonsteroidal anti-inflammatory drugs

\section{STANDARD TECHNIQUES FOR THERMAL HEMOSTATIC THERAPY}

The technique for endoscopic hemostatic therapy depends on the stigmata of ulcer hemorrhage and the experience of the endoscopists. The CURE Hemostasis Group's standardized techniques have changed because combination therapy (adrenalin injection and thermal therapy) appears to be significantly better than monotherapy (thermal therapy alone with bipolar probe or heater probe) for active arterial bleeding (Hong Kong and CURE trials [4,5]) and for nonbleeding adherent clots (Mayo [6] and CURE [7] trials). With current sample sizes, a significant difference between thermal therapy (gold probe) and combination therapy (injection plus gold probe) for nonbleeding visible vessels has not been shown in one trial (CURE). The goal of controlling active arterial bleeding is more often achieved by four quadrant injections of adrenalin $(1: 10,000$ concentration) in 0.5 to

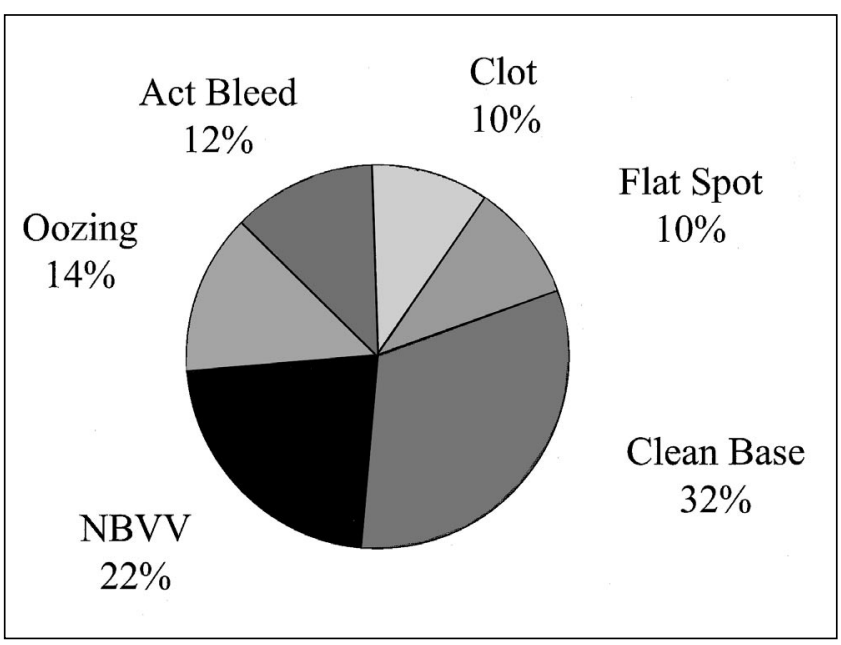

Figure 2) Endoscopic appearance of ulcer during or after severe ulcer hemorrhage. NBVV Nonbleeding visible vessel

TABLE 3

CURE Hemostasis Group definition of adherent clot on ulcer

- Amorphous red clot obscuring underlying stigmata

- Hooked onto ulcer base by pedicle

- Resistant to moderate target irrigation of water

- Distinguishable from a visible vessel by larger size and form

Data from references $1,2,7$

$1.0 \mathrm{~mL}$ aliquots, then coagulation with large diameter (10 French) gold probe directly on the bleeding point, tamponading it with firm pressure until the bleeding stops, then coagulating with long pulses ( 7 to $10 \mathrm{~s}$ in duration) at low power settings ( 15 to $20 \mathrm{~W}$ ). Washing, suctioning and repositioning to tamponade the bleeding point facilitate complete control of the bleeding and flattening of the visible vessel, if present (1). As many pulses as necessary to control active bleeding are used. Four quadrant adrenalin injections can be repeated if bleeding is not well controlled with thermal coagulation. Heater probe (10 French) with five to six pulses of $30 \mathrm{~J}$ each per tamponade station (delivering 150 to $180 \mathrm{~J}$ ) before moving the probe tip is also very effective for hemostasis of actively bleeding ulcers $(1,2)$.

Among endoscopists and investigators, it is important to standardize the diagnosis for an adherent clot. The CURE hemostasis group uses the criteria listed in Table 3. Distinguishing an adherent clot from a nonbleeding visible vessel is also critical (Table 4).

For the nonbleeding visible vessel, coagulation with either gold probe or heater probe directly on the visible vessel, with the same techniques as those used to treat the active arterial bleeder, flattens the visible vessel, usually coagulates the underlying vessel well and significantly reduces rebleeding rates compared with medical therapy alone (1). Table 5 summarizes the management of nonbleeding visible vessels.

For the adherent, nonbleeding clot on an ulcer that obscures the underlying stigmata, the standard CURE hemostasis team approach is as follows: four quadrant adrenalin 
TABLE 4

Management of a nonbleeding visible vessel versus an adherent clot on ulcer

\begin{tabular}{lcc}
\hline & $\begin{array}{c}\text { Nonbleeding visible } \\
\text { vessel }\end{array}$ & Adherent clot \\
\hline Size & Small $(\mathrm{mm})$ & Large $(\mathrm{cm})$ \\
Colour & Variable & Red \\
Form & Discrete & Amorphous \\
Relation to stigmata & Is stigma & Obscures stigma \\
$\begin{array}{l}\text { Rebleeding rate with } \\
\text { medical therapy }\end{array}$ & $50 \%$ & $30 \%$ \\
\hline
\end{tabular}

Data from references $1,2,7$

injection followed by cold guillotining off the clot to reveal the underlying stigmata, if the clot cannot be washed or suctioned off first, and, finally, coagulation of the underlying visible vessel with thermal therapy (7). In a CURE multicentre trial, this combination therapy improved the outcomes of patients compared with medical therapy, whereas a previous trial demonstrated similar outcomes of three monotherapies - medical therapy alone, heater probe and injection (adrenalin, then alcohol). Table 6 summarizes the management of adherent clots.

\section{USING THE CLINICAL CONDITION OF THE PATIENT AND THE ENDOSCOPIC APPEARANCE OF THE ULCER TO DETERMINE SUBSEQUENT LEVEL OF CARE}

In the tertiary care hospitals of the CURE Hemostasis Group, the clinical condition of the patient with upper gastrointestinal bleeding is used to determine whether patients should have continued hospitalization (in the intensive care unit, monitored bed or ward) or be followed up as an outpatient after endoscopy. The initial level of care is determined by clinical signs and assessment, such as the estimated severity of blood loss and consequent need for early resuscitation, determination of whether there is ongoing gastrointestinal bleeding or active gastrointestinal bleeding severe enough to warrant intensive care, and assessment of risk of the patient because of severe or unstable medical conditions (1-3). Because the CURE Hemostasis Group's patient population is predominantly elderly people (older than 65 years of age), with a high prevalence of active or severe comorbid conditions, urgent endoscopy and stigmata of hemorrhage are not used to determine the initial level of care. However, endoscopic findings after admission are used to plan subsequent care after initial resuscitation and stabilization of comorbid medical conditions.

Patients who may be candidates for early endoscopy and triage to early discharge or ward hospitalization $(8,9)$ are those who have less severe upper gastrointestinal hemorrhage, have stopped bleeding, have no significant comorbid conditions, are reliable and do not have significant hypovolemia. Patients with clean ulcer bases, flat spots or grey slough on endoscopy have very low rebleeding rates with medical therapy and, if they are reliable and able to return
TABLE 5

Management of nonbleeding visible vessel after severe ulcer bleeding

- The prevalence of a nonbleeding visible vessel after severe upper gastrointestinal hemorrhage is approximately $25 \%$

- The rebleeding rate is $40 \%$ to $50 \%$ on medical therapy

- Endoscopic therapy reduces the rebleeding rate to $15 \%$ to $20 \%$

- A biopsy for Helicobacter pylori is performed, a proton pump inhibitor is given and a regular diet is maintained

- The patient is retreated endoscopically for severe rebleeding

- The patient is observed for $48 \mathrm{~h}$ and discharged

\section{TABLE 6}

Management of an adherent clot on ulcer in a high risk patient after a severe upper gastrointestinal hemorrhage

- The prevalence of an adherent clot is approximately $10 \%$ for severe upper gastrointestinal hemorrhage

- The rebleeding rate is $30 \%$ to $40 \%$ with medical therapy

- Monotherapies do not reduce the rebleeding rate

- Adrenalin injection, washing, cold guillotining of clot, and bipolar or heater probe coagulation reduce the rebleeding rate by $<5 \%$

- The patient is biopsied for Helicobacter pylori and fed

for outpatient follow-up, are reasonable candidates for outpatient management of their ulcer hemorrhage $(8,9)$.

\section{REFERENCES}

1. Jensen DM. Endoscopic control of non-variceal upper gastrointestinal hemorrhage. In: Yamada T, Alpers D, Owyang C, Powell O, Silverstein F, eds. Textbook of Gastroenterology, 2nd edn. Philadelphia: JB Lippincott Co, 1995:2991-3011.

2. Kovacs TOG, Jensen DM. Therapeutic endoscopy in upper gastrointestinal bleeding. In: Tayler MB, Gollan JL, Steer ML, Wolfe MM, eds. Gastrointestinal Emergencies, 2nd edn. Philadelphia: Williams and Wilkens, 1997:181-98.

3. Cook DJ, Guyatt GH, Salena BJ, Laine L. Endoscopic therapy for acute non-variceal upper gastrointestinal hemorrhage: A meta-analysis. Gastroenterology 1992;102:139-48.

4. Chung SCS, Sung JY, Lai CW, Ng EKW, Chan KL, Yung MY. Epinephrine injection alone or epinephrine plus heat probe treatment for bleeding peptic ulcers: a randomized trial. Gastrointest Endosc 1994:40:271. (Abst)

5. Jensen DM, Kovacs T, Randall G, Smith J, Freeman M, Jutabha R. Prospective study of thermal coagulation (gold probe) versus combination injection and thermal treatment of high risk patients with severe ulcer or Mallory Weiss bleeding. Gastrointest Endosc 1994;40:25. (Abst)

6. Bleu BF, Gostout CJ, Shaw MJ, et al. Final results: Rebleeding from peptic ulcers associated with adherent clots: a prospective randomized controlled study comparing endoscopic therapy with medical therapy. Gastrointest Endosc 1997;45:251. (Abst)

7. Jensen DM, Kovacs TOG, Jutabha R, et al. Initial results of a multicenter, randomized controlled trial of medical versus combination endoscopic therapy for prevention of recurrent severe ulcer hemorrhage from non-bleeding adherent clots. Gastrointest Endosc 1996;43:352. (Abst)

8. Longstreth GF, Freitelberg SP. Outpatient care of selected patients with acute non-variceal upper gastrointestinal haemorrhage. Lancet 1995;345:108-11.

9. Lai KC, Hui WM, Wong BC, Ching CK, Lam SK. A retrospective and prospective study on the safety of discharging selected patients with duodenal ulcer bleeding on the same day as endoscopy. Gastrointest Endosc 1997;45:26-30. 


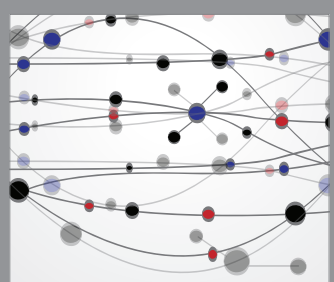

The Scientific World Journal
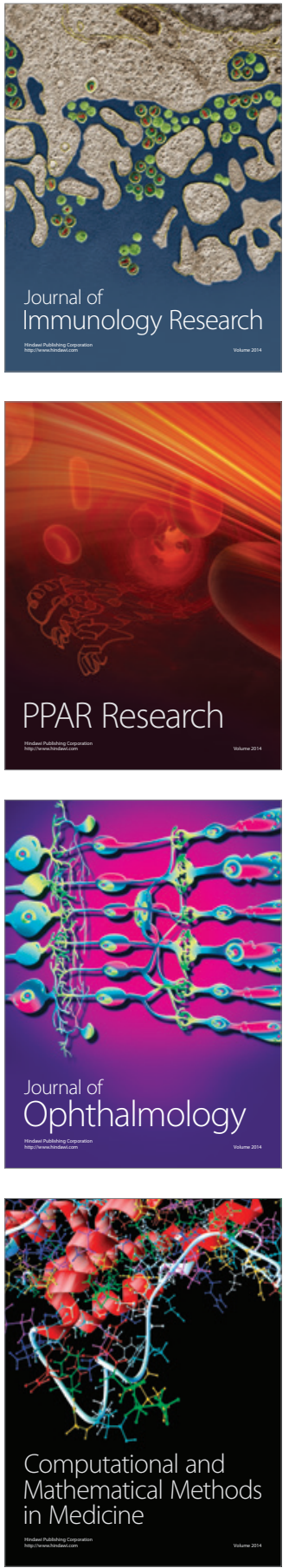

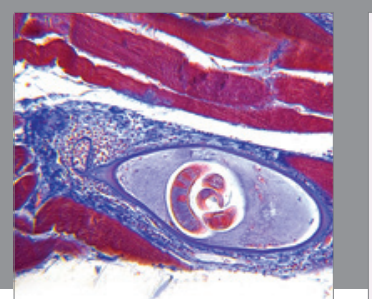

Gastroenterology Research and Practice

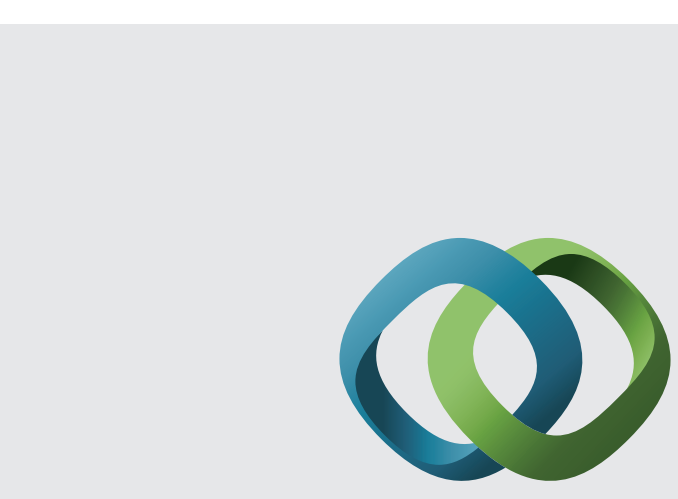

\section{Hindawi}

Submit your manuscripts at

http://www.hindawi.com
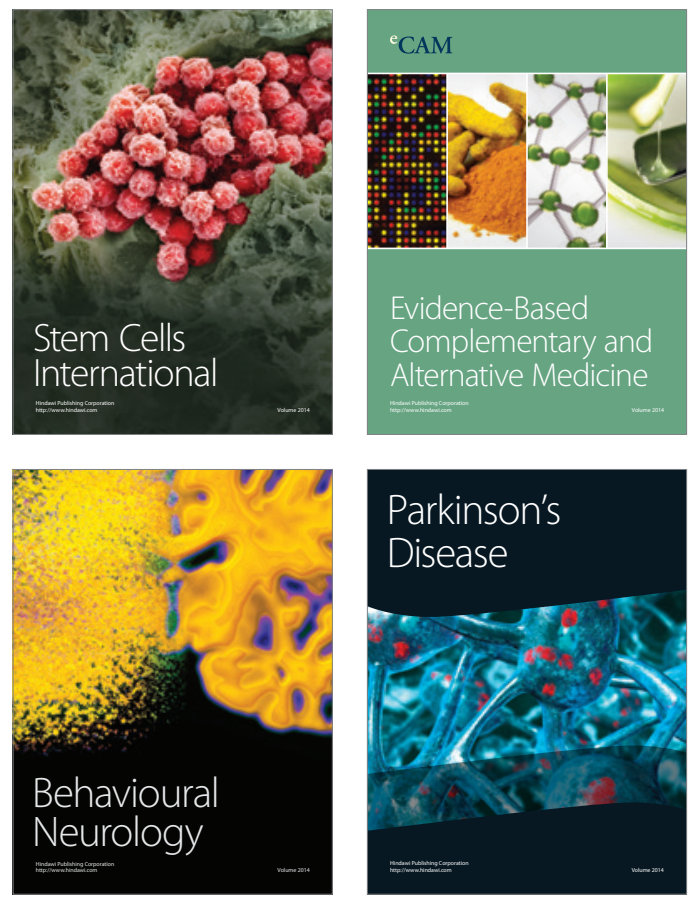
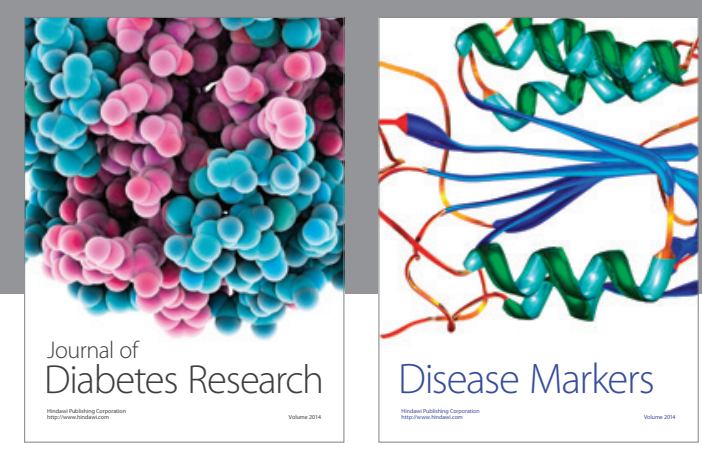

Disease Markers
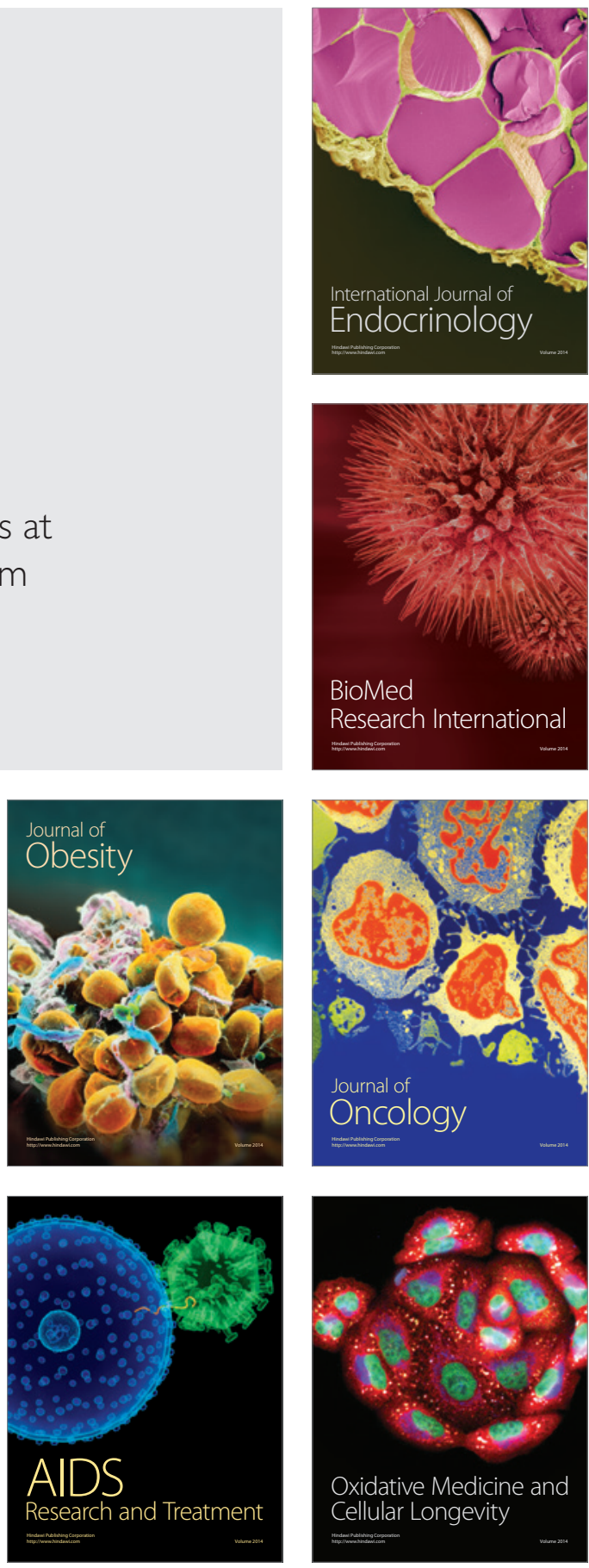